\title{
Impaired Smooth Pursuit During Transient Global Amnesia
}

\author{
Sung-Hee Kim ${ }^{a}$ \\ Young Ho Park \\ SangYun Kim ${ }^{\mathrm{b}}$ \\ Ji-Soo Kim ${ }^{\mathrm{b}}$ \\ aDepartment of Neurology, \\ School of Medicine, \\ Kyungpook National University, \\ Kyungpook National University \\ Chilgok Hospital, Daegu, Korea \\ ${ }^{b}$ Department of Neurology, \\ Seoul National University \\ College of Medicine, \\ Seoul National University \\ Bundang Hospital, Seoul, Korea
}

Background and Purpose During transient global amnesia (TGA), selective impairment of episodic memory is assumed to occur due to alteration in the neuronal network between the hippocampus and parietooccipital cortices that also include a hub for smooth pursuit (SP) eye movements. This study aimed to determine whether SP is impaired during TGA, and to identify any anatomical and functional linkage present between the oculomotor and memory systems.

Methods Within a median of 1.0 day of TGA, horizontal SP was evaluated in 145 patients with a target moving at peak velocities of $10 \%$ and $20 \%$. The average SP gains of patients were compared with those of the age-matched controls.

Results The patients with TGA showed lower SP gains in both directions for both peak target velocities. While the normal controls showed symmetric SP in the rightward and leftward directions, in the TGA patients the SP gain was lower during rightward than leftward SP regardless of bilaterality or the side of the lesions.

Conclusions The cortical regions processing information about visual motion appeared to be affected during or soon after an amnestic episode of TGA, and more so in the right hemisphere. This means that disturbed processing of dynamic visual information may be related to the impaired spatial orientation observed during TGA.

Key Words smooth pursuit, transient global amnesia, hippocampus.
Received September 19, 2018

Revised December 27, 2018

Accepted December 27, 2018

\section{Correspondence}

Ji-Soo Kim, MD, PhD

Department of Neurology,

Seoul National University

College of Medicine,

Seoul National University

Bundang Hospital,

82 Gumi-ro 173beon-gil, Bundang-gu,

Seongnam 13620, Korea

Tel $+82-31-787-7461$

Fax +82-31-719-6828

E-mail jisookim@snu.ac.kr

\section{INTRODUCTION}

Transient global amnesia (TGA) refers to an amnestic syndrome characterized by the sudden loss of anterograde and retrograde memory that lasts for up to 24 hours without other neurological deficits. ${ }^{1}$ Given that TGA elicits the selective loss of episodic memory, this condition may serve as a model for the neurological disorders affecting the CA1 sector of the hippocampal cornu ammonis. ${ }^{2}$ Two parallel connections between the hippocampus and neocortex have been proposed: the anterior temporal and posterior medial networks. ${ }^{3}$ While the perirhinal cortex is closely connected with the anterior temporal and frontal cortical areas, the parahippocampal cortex is connected with the posterior medial temporal, parietal, and occipital areas. ${ }^{4}$ Although functional differentiation between the anterior and posterior hippocampi remains controversial, episodic memory appears to involve the posterior medial network more than the anterior temporal network. ${ }^{5}$ Hippocampal atrophy alone seems to be insufficient to cause episodic memory impairments, even in neurodegenerative diseases. ${ }^{6}$ Indeed, episodic memory is impaired during disruption of the posterior medial network, which connects the hippocampus and the posterior cortical areas. ${ }^{6}$ Given that patients with TGA show markedly impaired episodic memory, the posterior medial network may be affected primarily during TGA. ${ }^{7}$ A study using a spectral EEG analysis found an alteration in neuronal activity in the cortical regions involving the pos-

(a) This is an Open Access article distributed under the terms of the Creative Commons Attribution Non-Commercial License (https://creativecommons.org/licenses/by-nc/4.0) which permits unrestricted non-commercial use, distribution, and reproduction in any medium, provided the original work is properly cited. 
terior medial network during TGA. ${ }^{8}$

Smooth pursuit (SP) occurs when observing a slowly moving object of interest and enables the continuous maintenance of clear vision. The posterior parietal cortex is known to be important for $\mathrm{SP}^{9}$ and unilateral lesions involving this area in humans impair SP, more so toward the lesion side. ${ }^{10}$ We therefore hypothesized that if the amnesia during TGA is caused by alteration in the neuronal network between the hippocampus and parietooccipital cortices, these patients may show impaired SP due to dysfunction of the SP pathways located in the parietooccipital cortices. The present study aimed to determine whether SP is impaired during TGA and to identify any anatomical and functional linkage present between the oculomotor and memory systems.

\section{METHODS}

\section{Participants}

This study included 145 patients (99 women) who arrived at the hospital within 7 days of symptom onset and fulfilled the following diagnostic criteria of TGA ${ }^{1}$ : 1) Attack must have been witnessed by a capable observer, with information about it available over most of its duration, 2) Clear-cut anterograde amnesia during the attack, 3) No clouding of consciousness or loss of personal identity, and cognitive impairment limited to amnesia, 4) No focal neurological symptom during the attack and no significant neurological sign thereafter, 5) No epileptic features, 6) Attack resolved within 24 hours, and 7) No recent head injury or active epilepsy. We also excluded the patients taking medication known to affect SP, such as hypnotics and benzodiazepines.

The study was performed in accordance with the Declaration of Helsinki and approved by the local Institutional Review Board (IRB No. B-1809-492-103).

\section{Evaluation of smooth pursuit}

Eye motion was recorded using video-oculography (VOG) with a resolution of $0.1^{\circ}$ and a sampling rate of $60 \mathrm{~Hz}$ in all 145 patients. VOG was performed within a median of 1.0 day from the onset of TGA (range 0 to 7 days) (Table 1). The stimulus for SP was an illuminated red target moving along a sinusoidal trajectory at peak velocities of $10 \%$ and $20 \%$ s. Participants were seated $110 \mathrm{~cm}$ from the target and instructed to look at it without moving their head. Eye and target velocities were obtained by two-point digital differentiation. After eliminating saccadic eye movements detected based on a velocity threshold criterion $(100 \%)$, the SP gain was determined as the ratio of the peak eye velocity to the peak target velocity (PTV). A linear interpolation method was used to bridge the gaps produced by removing saccades. The av-
Table 1. Clinical features of the 145 patients with TGA

\begin{tabular}{lc}
\hline & $\boldsymbol{n}(\%)$ \\
\hline Age, years & $59.2 \pm 9.0$ \\
Sex, women & $99(68.2)$ \\
Duration of TGA, hours & $6.7 \pm 5.9$ \\
Precipitating factor & \\
$\quad$ Physical stress & $19(13.1)$ \\
\hline Emotional stress & $39(26.8)$ \\
Associated symptom & \\
\hline Dizziness, nausea, or vomiting & $7(18.6)$ \\
$\quad$ Headache & $28(19.3)$ \\
Hypertension & $48(33.1)$ \\
Diabetes & $9(6.2)$ \\
Dyslipidemia & $33(22.7)$ \\
Laterality of DWI lesion & \\
$\quad$ Right & $42(29.5)$ \\
\hline Left & $44(30.9)$ \\
\hline Bilateral & $36(25.3)$ \\
\hline Location of DWI lesion* & \\
\hline Head & $48(33.8)$ \\
\hline Body & $89(62.6)$ \\
\hline Tail & $13(9.1)$ \\
\hline Treaterval between symptom onset and VOG, days & $1.0,1.0$ \\
\hline Data antiplatelet drugs & $14(9.8)$ \\
\hline
\end{tabular}

Data are mean $\pm S D, n(\%)$, or median, IOD values.

*Brain MRI was performed in 142 patients with TGA, of whom 122 showed a 1- to 5-mm punctate high-signal-intensity lesion in the hippocampus in DWI. A single lesion involving the head, body, or tail of the hippocampus was present in 94 patients, while 28 patients showed multiple lesions.

IQD: interquartile distance, TGA: transient global amnesia, VOG: videooculography.

erage SP gain of patients was compared with the value obtained in 50 normal volunteers without dizziness or vertigo. Since SP gain decreases with age and the normal controls began to show a significant difference at an age of 50 years, the participants were divided into younger and older groups using the mean age of 50 years as the cutoff (Table 2). We calculated the SP asymmetry as (rightward gain-leftward gain)/ (rightward gain+leftward gain) $\times 100 \%$.

\section{Brain MRI}

MRI was performed in 142 patients with TGA and comprised DWI, T1- and T2-weighted imaging, FLAIR imaging, and conventional gradient-echo imaging in the transverse plane, three-dimensional time-of-flight angiography of the intracranial region, and contrast-enhanced angiography of the neck region. We also performed subsequent thin-section DWI and T2-weighted imaging in the coronal planes that were perpendicular to the long axis of the hippocampus. 
Table 2. Comparison between the younger and older patients with TGA

\begin{tabular}{lrccc}
\hline \multicolumn{1}{c}{ All patients } & Women & Age, years & Interval between symptom onset and VOG, days & Dizziness, nausea, or vomiting \\
\hline Total, $n=145$ & $99(68.2)$ & $59.2 \pm 9.0$ & $1.0,1.0$ & $27(18.6)$ \\
Younger group, $n=17$ & $9(52.9)$ & $44.1 \pm 7.7$ & $2.0,1.5$ & $4(23.5)$ \\
Older group, $n=128$ & $90(70.3)$ & $61.3 \pm 7.0$ & $1.0,1.0$ & $23(17.9)$ \\
\hline
\end{tabular}

Data are mean \pm SD, $n(\%)$, or median, IQD values. Patients were divided into younger and older groups using the mean age of 50 years as the cutoff. IOD: interquartile distance, TGA: transient global amnesia, VOG: video-oculography.

\section{Statistical analysis}

The data for variables that conformed to a Gaussian distribution, such as age and SP gain, are presented as mean and SD values. The differences in the average SP gain between patients and age-matched controls were analyzed using $t$-tests, and those between rightward and leftward SP were analyzed using paired $t$-tests. Since the interval between the VOG evaluation and the symptom onset as well as the asymmetry of SP gain did not conform to a Gaussian distribution, the data for these variables are presented as median, interquartile distance (IQD), and extreme values. SP gain asymmetry was compared between the patients and age-matched controls using the Mann-Whitney test. Probability values of $p<0.05$ were considered statistically significant.

\section{RESULTS}

\section{Clinical findings}

The clinical characteristics of the study population are summarized in Table 1 . All of the 145 patients with TGA exhibited a full range of eye movements and normal visual fields. Twenty-seven patients (18.6\%) reported the symptom of dizziness, nausea, or vomiting. Nineteen (13.1\%) patients showed spontaneous nystagmus: nine with horizontal, six with verti$\mathrm{cal}$, and four with mixed horizontal-vertical nystagmus. Twenty-three (15.8\%) patients showed saccadic abnormalities. Hypermetria was noted in 12 (8.2\%) patients: rightward in 6, leftward in 1, and bilaterally in 5 . Hypometria was noted in $11(7.5 \%)$ patients: rightward in 5 , leftward in 4 , and bilaterally in 2 .

\section{MRI}

In $122(85.9 \%)$ of the 142 patients in whom MRI was performed, DWI disclosed a 1- to 5-mm punctate high-signalintensity lesion in the hippocampus, which matched a small area of low signal intensity on apparent diffusion coefficient maps. The lesions were bilateral in 36 (25.3\%) of the $142 \mathrm{pa}-$ tients, while the $86(60.5 \%)$ patients with unilateral lesions comprised $42(29.5 \%)$ with those on the right side and 44 (30.9\%) on the left side (Table 1).

\section{Smooth pursuit}

Among younger controls ( $n=30$, age $=34.9 \pm 8.0$ years), the SP gains were $0.83 \pm 0.05$ and $0.82 \pm 0.04$ at PTVs of $10 \%$ and $20 \%$, respectively. When compared with the controls, the younger patients with TGA ( $n=17$, age $=44.1 \pm 7.7$ years) showed lower SP gains in both directions: $0.68 \pm 0.13$ and $0.65 \pm 0.18$ at PTVs of $10 \%$ and $20 \%$, respectively (both $p<$ 0.001 ) (Fig. 1). Among older controls ( $n=20$, age $=60.3 \pm 6.7$ years), the SP gains were $0.78 \pm 0.09$ and $0.74 \pm 0.10$ at PTVs of $10 \%$ and $20 \%$, respectively. Compared with the age-matched controls, the older patients with TGA $(n=132$, age $=61.3 \pm 7.0$ years) showed lower SP gains in both directions regardless of the PTV: $0.61 \pm 0.14$ and $0.55 \pm 0.17$ at PTVs of $10 \%$ and $20 \%$, respectively (both $p<0.001$ ) (Fig. 1 ).

In the controls, the horizontal SP was symmetric in the rightward and leftward directions $(p=0.374$ and $p=0.622$ at PTVs of $10 \%$ and $20 \%$, respectively). The median asymmetry among the younger controls was $1.8 \%(\mathrm{IQD}=2.9 \%)$ at a PTV of $10 \%$ and $2.1 \%(\mathrm{IQD}=2.3 \%)$ at a PTV of $20 \% \mathrm{~s}(\mathrm{Ta}-$ ble 3). The median asymmetry among the older controls was $2.7 \%(\mathrm{IQD}=2.7 \%)$ at a PTV of $10 \%$ and $2.4 \%(\mathrm{IQD}=3.6 \%)$ at a PTV of $20 \%$. In contrast, the TGA patients showed asymmetric SP gains between the rightward and leftward directions ( $p<0.001$ and $p=0.026$ at PTVs of $10 \%$ and $20 \%$, respectively). This asymmetry of the horizontal SP was significantly greater in the TGA patients than the controls (Table 3), and greater in the older patients when the target moved faster: $7.4 \%(\mathrm{IQD}=7.9 \%, p<0.001)$ at a PTV of $10 \%$ and $9.4 \%$ (IQD= $14.5 \%, p<0.001)$ at a PTV of $20 \%$ s.

The TGA patients who had no discernible hippocampal lesions in DWI showed comparatively symmetric SP gains. In contrast, irrespective of the unilaterality and side of the MRI lesions, the SP gain was lower on the right than the left among the patients (Fig. 2). The difference between rightward and leftward SP gains was significant in the patients with leftsided or bilateral lesions when tested at the lower velocity of target motion (Table 4).

\section{DISCUSSION}

Smooth visual tracking ensures that a slowly moving target can be visualized clearly by holding its retinal image within 
the fovea. Since the visual acuity is markedly lower for a visual target whose retinal image is outside the fovea, SP is driven by signals from the cortical areas that process information about visual motion containing the direction and velocity of an object dislocated from the center of the fovea. ${ }^{11}$ In monkeys, the middle temporal (MT) and medial superior temporal (MST) areas within the superior temporal sulcus form the primary hub for processing information about visual mo-
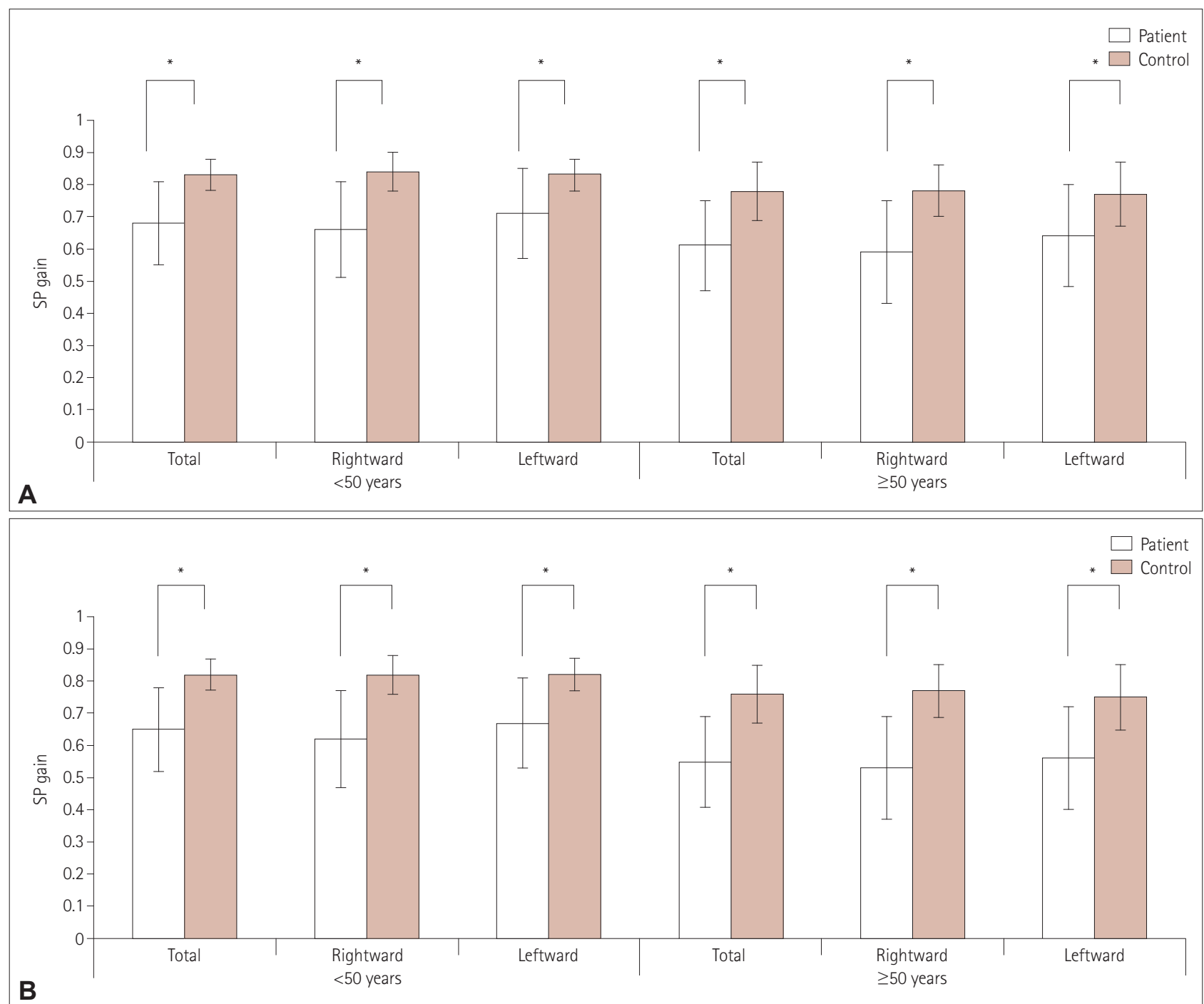

Fig. 1. Comparison of SP gains between the patients with TGA and controls. Compared to the age-matched controls, the patients showed lower SP gains in both directions when tested with a target moving sinusoidally at PTVs of $10 \% \mathrm{~s}(\mathrm{~A})$ and $20 \% \mathrm{~s}(\mathrm{~B})$. In younger controls (age $=34.9 \pm 8.0$ years), the SP gains were $0.83 \pm 0.05$ and $0.82 \pm 0.04$ at PTVs of $10 \%$ s and $20 \% \mathrm{~s}$, respectively. When compared with controls, the younger patients with TGA (age $=44.1 \pm 7.7$ years) showed lower SP gains in both directions: $0.68 \pm 0.13$ at a PTV of $10^{\circ} / \mathrm{s}$ and $0.65 \pm 0.18$ at a PTV of $20^{\circ} / \mathrm{s}$ (both $p=0.001$ ). Among older controls (age $=60.3 \pm 6.7$ years), the SP gains were $0.78 \pm 0.09$ and $0.74 \pm 0.10$ at PTVs of $10 \%$ s and $20 \%$, respectively. Compared with the age-matched controls, the older patients with TGA (age $=61.3 \pm 7.0$ years) showed lower SP gains in both directions regardless of the PTV: $0.61 \pm 0.14$ at $10^{\circ} / \mathrm{s}$ and $0.55 \pm 0.17$ at $20 \%$ (both $p<0.001$ ). ${ }^{*} p<0.01$ in t-tests. PTV: peak target velocity, SP: smooth pursuit, TGA: transient global amnesia.

Table 3. Comparison of SP asymmetry between patients with TGA and controls

\begin{tabular}{|c|c|c|c|c|c|c|}
\hline \multirow{2}{*}{ Age, years } & \multicolumn{3}{|c|}{ Asymmetry of SP gain at a PTV of $10 \% / \mathrm{s}(\%)$} & \multicolumn{3}{|c|}{ Asymmetry of SP gain at a PTV of $20 \% / \mathrm{s}(\%)$} \\
\hline & Patients & Controls & $p$ & Patients & Controls & $p$ \\
\hline$<50$ & $5.7,9.9$ & $1.8,2.9$ & $<0.001$ & $5.1,6.6$ & $2.1,2.3$ & $<0.001$ \\
\hline$>50$ & $7.4,7.9$ & $2.7,2.7$ & $<0.001$ & $9.4,14.5$ & $2.4,3.6$ & $<0.001$ \\
\hline
\end{tabular}

Data are median, IQD values. SP gain asymmetry was compared between the patients and controls using the Mann-Whitney test.

IOD: interquartile distance, PTV: peak target velocity, SP: smooth pursuit, TGA: transient global amnesia. 


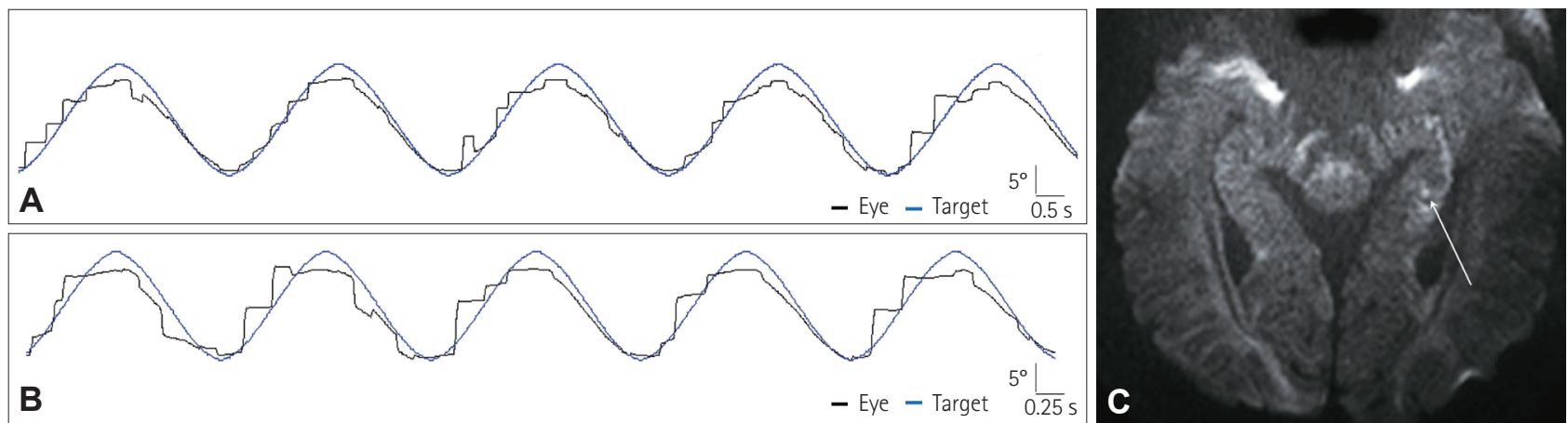

Fig. 2. A representative case of transient global amnesia. A 46-year-old woman showed saccadic smooth pursuit mostly to the right when tested with a target moving sinusoidally with peak target velocity of $10 \%$ (A, rightward gain of 0.32 and leftward gain of 0.69 ; asymmetry of $36.6 \%)$ and $20 \% \mathrm{~s}$ (B, rightward gain of 0.19 and leftward gain of 0.56; asymmetry of 49.3\%). Brain MRI showed a punctate high-signal-intensity lesion in the left hippocampal body (C, arrow).

Table 4. SP gain according to hippocampal lesions in MRI

\begin{tabular}{|c|c|c|c|c|c|c|c|c|}
\hline \multirow{2}{*}{ MRI lesion } & \multicolumn{4}{|c|}{ SP gain at a PTV of $10^{\circ} / \mathrm{s}$} & \multicolumn{4}{|c|}{ SP gain at a PTV of $20^{\circ} / \mathrm{s}$} \\
\hline & Rightward & Leftward & $p$ & Asymmetry (\%) & Rightward & Leftward & $p$ & Asymmetry (\%) \\
\hline Right & $0.60 \pm 0.15$ & $0.64 \pm 0.18$ & 0.056 & $7.6,11.9$ & $0.54 \pm 0.19$ & $0.56 \pm 0.21$ & 0.588 & $8.2,11.7$ \\
\hline Left & $0.56 \pm 0.17$ & $0.63 \pm 0.14$ & 0.007 & $8.0,10.1$ & $0.50 \pm 0.22$ & $0.56 \pm 0.16$ & 0.060 & $10.6,24.8$ \\
\hline Bilateral & $0.60 \pm 0.16$ & $0.68 \pm 0.15$ & 0.001 & $7.4,6.1$ & $0.57 \pm 0.19$ & $0.62 \pm 0.19$ & 0.052 & $9.0,13.7$ \\
\hline None & $0.63 \pm 0.14$ & $0.64 \pm 0.13$ & 0.825 & $5.0,6.4$ & $0.58 \pm 0.15$ & $0.56 \pm 0.20$ & 0.383 & $8.1,14.1$ \\
\hline
\end{tabular}

Data are mean \pm SD or median, IOD values.

Differences in the mean gains of SP between rightward and leftward SP were analyzed using paired $t$-tests.

IOD: interquartile distance, PTV: peak target velocity, SP: smooth pursuit.

tion. ${ }^{12}$ The neurons in the MT and MST areas exhibit direction and velocity selectivity, ${ }^{13}$ and damage to these areas causes defective SP. ${ }^{14}$ The human homologues of the MT and MST areas are assumed to lie adjacent to the occipitotemporoparietal junction involving Brodmann areas 19 and $39 .{ }^{15}$ These cortical regions belong to the posterior medial memory network that appears to be affected during TGA, ${ }^{8}$ and this anatomical linkage might explain why the SP gain was significantly lower in our patients with TGA than in the controls.

SP requires a combination of multilevel activities including visual motion perception/processing, sensorimotor transformation, and cognitive processes such as attention and execution. ${ }^{11,16}$ Attention is important to the ability to perform SP. ${ }^{17,18}$ Previous studies support that SP and attentional processes share neural substrates, including the frontal eye fields, supplementary eye fields, and prefrontal cortex. ${ }^{19,20}$ Although attention may affect the SP performance in patients with TGA, a global attentional problem alone cannot explain the asymmetry in the rightward and leftward SP observed in our patients with TGA. Such significant SP asymmetry supports that deficits of SP during TGA are caused by lateralized abnormalities of the SP pathways. A study of seven TGA patients using SPECT indeed showed decreased blood flows in the inferior and middle frontal gyri bilaterally, but the reductions were greater on the left side in the superior temporal, precentral, and postcentral gyri. ${ }^{21}$ Other studies have also revealed impaired perfusion in the left hemisphere during TGA attacks, ${ }^{22,23}$ suggesting that lateralized brain dysfunction is an important component of TGA pathophysiology. These hemisphere-specific disturbances of TGA may contribute to dysfunction of the cerebrocortical pathways involved in the control of SP, and cause asymmetric impairment of SP. It is particularly notable that our patients with TGA showed bilaterally impaired SP that was greater on the right irrespective of the lesion side. Given that the afferent visual pathways and the initial stages of cortical visual processing represent a retinotopic organization of the contralateral visual space, unilateral lesions of the geniculostriate visual pathway can barely induce asymmetric impairment of $\mathrm{SP}^{24}$ Any visual inattention that is usually associated with nondominant parietooccipital damage is also unlikely to cause an asymmetric pursuit defect, since neglect to the contralateral space with a reference to head position usually causes a symmetric pursuit deficit in the contralateral hemifield. ${ }^{10}$ Dysfunction of the parietooccipital cortices or their corticofugal projections decreases the pursuit gain more toward the lesion side in humans, even though cerebral control of SP is not strictly ipsilateral. ${ }^{10}$ Thus, the greater SP impairment toward the right in our patients suggests that the cortical regions processing the information about visual motion were more affected on the right side during or soon 
after the amnestic episode. Since the present study did not include follow-up oculography, further investigations are required to determine whether this asymmetric impairment of horizontal SP found during or just after amnesia is a transient or longer lasting phenomenon. Moreover, since our study did not evaluate the vestibulo-ocular reflex using a head impulse or bithermal caloric test, the current results cannot exclude the effects of vestibular asymmetry.

The velocity of SP ideally matches that of the moving target, which corresponds to an SP gain of 1.0. However, the SP gain can readily vary even during optimal SP performance due to the innate dynamic properties of the SP system. ${ }^{25}$ Maintaining SP hence requires complex neuronal activity including prediction of the target velocity and continuous visual feedback about the ongoing SP performance. A complex neural network-such as the dorsal cortical eye fields and cerebellum-is involved in achieving an optimal SP response by integrating extraretinal information. ${ }^{26}$ The cerebellum provides the principal feedback role in the SP system. ${ }^{27}$ A study of the correlation between SPECT and MRI findings also found a predominant hypoperfusion in the cerebellar vermis in combination with punctuate DWI lesions in the hippocampus in five patients with TGA during the time window of 24 to 73 hours after onset. ${ }^{28}$ Twelve of our patients indeed showed the saccadic hypermetria that is a distinctive ocular motor feature of cerebellar dysfunction. Given that the cerebellar vermis is closely connected to the MT and MST areas via the pontine nuclei, ${ }^{29}$ the occurrence of cerebellar dysfunction during a TGA attack might be linked to the disruption of the posterior cerebral hemispheric network that was found in our patients.

The visual motion cues that drive SP are essential for object detection, visuospatial perception, and navigation during locomotion. Cognitive function assessment and functional MRI in a patient with TGA showed a reduced or no activation of the temporal lobe structures during encoding of visual scenes or recognition of old scenes..$^{30}$ Perceiving and navigating visual scenes are basic abilities that we use when interacting with our environment, and reliable copies of our environment require an optimal visual acuity. Visual information about our surroundings is integrated with various other types of information via complex neural networks to establish concepts on how the self is related to the world. ${ }^{11}$ An impaired SP performance might be attributable to a diminished perception of the objects located in our surroundings and have an impact on visual information processing within the referential system, leading to impaired spatial orientation. This mechanism would provide a plausible explanation for why patients with acute TGA frequently show spatial learning and navigation deficits. ${ }^{30,31}$
Conflicts of Interest

The authors have no potential conflicts of interest to disclose.

\section{REFERENCES}

1. Hodges JR, Warlow CP. Syndromes of transient amnesia: towards a classification. A study of 153 cases. J Neurol Neurosurg Psychiatry 1990; 53:834-843.

2. Bartsch T, Deuschl G. Transient global amnesia: functional anatomy and clinical implications. Lancet Neurol 2010;9:205-214.

3. Burwell RD. The parahippocampal region: corticocortical connectivity. Ann N Y Acad Sci 2000;911:25-42.

4. Libby LA, Ekstrom AD, Ragland JD, Ranganath C. Differential connectivity of perirhinal and parahippocampal cortices within human hippocampal subregions revealed by high-resolution functional imaging. J Neurosci 2012;32:6550-6560.

5. Ranganath C, Ritchey M. Two cortical systems for memory-guided behaviour. Nat Rev Neurosci 2012;13:713-726.

6. La Joie R, Landeau B, Perrotin A, Bejanin A, Egret S, Pélerin A, et al. Intrinsic connectivity identifies the hippocampus as a main crossroad between Alzheimer's and semantic dementia-targeted networks. Neuron 2014;81:1417-1428.

7. Yi S, Park YH, Jang JW, Lim JS, Chun IK, Kim S. Decreased metabolism in the posterior medial network with concomitantly increased metabolism in the anterior temporal network during transient global amnesia. Brain Topogr 2018;31:468-476.

8. Park YH, Jeong HY, Jang JW, Park SY, Lim JS, Kim JY, et al. Disruption of the posterior medial network during the acute stage of transient global amnesia: a preliminary study. Clin EEG Neurosci 2016;47:69-74.

9. Trenner MU, Fahle M, Fasold O, Heekeren HR, Villringer A, Wenzel R. Human cortical areas involved in sustaining perceptual stability during smooth pursuit eye movements. Hum Brain Mapp 2008;29: 300-311.

10. Morrow MJ, Sharpe JA. Cerebral hemispheric localization of smooth pursuit asymmetry. Neurology 1990;40:284-292.

11. Lencer R, Trillenberg P. Neurophysiology and neuroanatomy of smooth pursuit in humans. Brain Cogn 2008;68:219-228.

12. Komatsu H, Wurtz RH. Modulation of pursuit eye movements by stimulation of cortical areas MT and MST. J Neurophysiol 1989;62:31-47.

13. Mikami A, Newsome WT, Wurtz RH. Motion selectivity in macaque visual cortex. I. Mechanisms of direction and speed selectivity in extrastriate area MT. J Neurophysiol 1986;55:1308-1327.

14. Dürsteler MR, Wurtz RH. Pursuit and optokinetic deficits following chemical lesions of cortical areas MT and MST. J Neurophysiol 1988; 60:940-965.

15. Orban GA, Van Essen D, Vanduffel W. Comparative mapping of higher visual areas in monkeys and humans. Trends Cogn Sci 2004;8:315324.

16. Barnes GR. Cognitive processes involved in smooth pursuit eye movements. Brain Cogn 2008;68:309-326.

17. Kerzel D, Souto D, Ziegler NE. Effects of attention shifts to stationary objects during steady-state smooth pursuit eye movements. Vision Res 2008;48:958-969.

18. Treue S, Maunsell JH. Effects of attention on the processing of motion in macaque middle temporal and medial superior temporal visual cortical areas. J Neurosci 1999;19:7591-7602.

19. Contreras R, Ghajar J, Bahar S, Suh M. Effect of cognitive load on eyetarget synchronization during smooth pursuit eye movement. Brain Res 2011;1398:55-63.

20. Hutton SB, Tegally D. The effects of dividing attention on smooth pursuit eye tracking. Exp Brain Res 2005;163:306-313.

21. Chung YA, Jeong J, Yang DW, Kang BJ, Kim SH, Chung SK, et al. A Tc-99m SPECT study of regional cerebral blood flow in patients with transient global amnesia. Neuroimage 2009;47:50-55. 
22. Kim BS, Cho SS, Choi JY, Kim YH. Transient global amnesia: a study with Tc-99m ECD SPECT shortly after symptom onset and after recovery. Diagn Interv Radiol 2016;22:476-480.

23. Matsuda H, Higashi S, Tsuji S, Sumiya H, Miyauchi T, Hisada K, et al. High resolution Tc-99m HMPAO SPECT in a patient with transient global amnesia. Clin Nucl Med 1993;18:46-49.

24. Segraves MA, Goldberg ME, Deng SY, Bruce CJ, Ungerleider LG, Mishkin $M$. The role of striate cortex in the guidance of eye movements in the monkey. J Neurosci 1987;7:3040-3058.

25. Robinson DA. The mechanics of human smooth pursuit eye movement. J Physiol 1965;180:569-591.

26. Ono S, Mustari MJ. Smooth pursuit-related information processing in frontal eye field neurons that project to the NRTP. Cereb Cortex 2009; 19:1186-1197.

27. Lindner A, Haarmeier T, Erb M, Grodd W, Thier P. Cerebrocerebellar circuits for the perceptual cancellation of eye-movement-induced retinal image motion. J Cogn Neurosci 2006;18:1899-1912.

28. Yang Y, Kim JS, Kim S, Kim YK, Kwak YT, Han IW. Cerebellar hypoperfusion during transient global amnesia: an MRI and oculographic study. J Clin Neurol 2009;5:74-80.

29. Keller EL, Heinen SJ. Generation of smooth-pursuit eye movements: neuronal mechanisms and pathways. Neurosci Res 1991;11:79-107.

30. Westmacott R, Silver FL, McAndrews MP. Understanding medial temporal activation in memory tasks: evidence from fMRI of encoding and recognition in a case of transient global amnesia. Hippocampus 2008; 18:317-325.

31. Bartsch T, Schönfeld R, Müller FJ, Alfke K, Leplow B, Aldenhoff J, et al. Focal lesions of human hippocampal CA1 neurons in transient global amnesia impair place memory. Science 2010;328:1412-1415. 